\title{
DOCENCIA, INVESTIGACIÓN Y EMPRESAS, VINCULADOS EN UN SISTEMA DE INFORMACIÓN
}

Hilda Beatriz Ramírez-Moreno: Universidad Autónoma de Baja California. México ramirezmb@uabc.edu.mx

Margarita Ramírez-Ramírez: Universidad Autónoma de Baja California. México. maguiram@uabc.edu.mx

\section{RESUMEN}

En la actualidad, las organizaciones son cada vez más complejas, cada día incorporan nuevas tecnologías a su forma de trabajar con lo que consiguen competir en el mercado globalizado que el mundo actualmente maneja. Por esta razón es importante que los Sistemas de Información proporcionen todos los datos necesarios para el proceso toma de decisiones, permitiendo de esta manera mejorar su forma de trabajo, logrando hacerse más competitivos. El Sistema Estatal de Información y Documentación Científica y Tecnológica de los Recursos Humanos, Materiales de Organización y Financieros, fue desarrollado para integrar la base para la generación de productos y servicios tecnológicos, logrando una divulgación y promoción del estado de Baja California. Este Sistema de Información aportara un panorama efectivo, eficiente y competitivo del desarrollo tecnológico de la región es imprescindible conocer las actividades de las Universidades, Empresas, Investigadores-Docentes, que cada uno de ellos está realizando, como son sus líneas de investigación, la productividad que tienen, investigaciones, todo esto con el objetivo de lograr unir esfuerzos en áreas comunes, manteniendo una relación de conocimiento, colaboración y apoyo.

PALABRAS CLAVE: Sistema de Información - SIEDCyT - Tecnología - Vinculación

\footnotetext{
${ }^{1}$ Autor correspondiente

Hilda Beatriz Ramírez-Moreno1: Universidad Autónoma de Baja California, Facultad de Contaduría y Administración, Tijuana B.C. México.

Correo: ramirezmb@uabc.edu.mx
} 


\title{
TEACHING, RESEARCH AND COMPANIES LINKED IN AN INFORMATION SYSTEM
}

\begin{abstract}
Today, organizations are increasingly complex, incorporating new technologies every day the way you work with what they get to compete in the global market currently operates the world. It is therefore important that information systems provide all the necessary data for decision-making process, thus allowing to improve the way work will get done more competitive. The State Information System and Information Science and Technology Human Resources, Organization and Financial Materials, was developed to integrate the basis for the generation of technology products and services, achieving dissemination and promotion of the state of Baja California. This system information provides an overview of effective, efficient and competitive technological development of the region is essential to know the activities of universities, companies, researchers and teachers, each of them is doing, as are its lines of research productivity with, research, all with the goal of uniting efforts in common areas, maintaining a relationship of knowledge, collaboration and support.
\end{abstract}

KEY WORDS: Information System - Siedcyt - Technology - Linking

\section{INTRODUCCIÓN}

En la actualidad, las organizaciones son cada vez más complejas, cada día incorporan nuevas tecnologías a su forma de trabajar con lo que consiguen competir en el mercado globalizado que el mundo actualmente maneja. Por esta razón es importante que los Sistemas de Información proporcionen todos los datos necesarios para el proceso toma de decisiones, permitiendo de esta manera mejorar su forma de trabajo, logrando hacerse más competitivos, por tal motivo es necesario comprender lo importante que representan los datos en las empresas, su almacenamiento y su uso posterior mediante el uso de las Tecnologías de Información. 
Las tecnologías de información son parte de nuestra sociedad han permitido el desarrollo de nuevos servicios, las empresas, las organizaciones y los gobiernos han logrado niveles nunca antes pensados en eficiencia, cobertura y eficacia. Dependemos de forma creciente de la ciencia y la tecnología; los procesos de producción, la medicina, la educación, la comunicación o el transporte son todos campos cuyo presente y futuro están ligeramente ligados al desarrollo tecnológico y científico, particularmente a la innovación.

El Sistema de Información "SIEDCyT" (Sistema Estatal de Información y Documentación Científica y Tecnológica de los Recursos Humanos, Materiales de Organización y Financieros), fue desarrollado para integrar la base para la generación de productos y servicios tecnológicos, logrando una divulgación y promoción del estado de Baja California. El sistema está diseñado para usos de diferentes niveles, esto es usuarios desde la Administración del sistema, usuarios de niveles de gestión y administración pública y usuarios finales.

El estado de Baja California tiene como uno de sus objetivos generales convertir a la ciencia, la tecnología y la innovación en los pilares de su crecimiento económico". El SIEDCyT es un Sistema de Información que aportara un panorama efectivo, eficiente y competitivo del desarrollo tecnológico de la región, es imprescindible conocer las actividades de las Universidades, Empresas, Investigadores-Docentes, que cada uno de ellos está realizando, como son sus líneas de investigación, la productividad que tienen, investigaciones, todo esto con el objetivo de lograr unir esfuerzos en áreas comunes, manteniendo una relación de conocimiento, colaboración y apoyo.

\section{METODOLOGÍA}

Las principales fuentes de información generadas por la investigación científica y tecnológica, centros de investigación e investigadores, se encuentran en el sector educativo. En este marco, el gobierno de Baja California decide trasladar el Consejo Estatal de Ciencia y Tecnología (COCyT), de la Secretaría de Educación y Bienestar Social a la SEDECO (Secretaria de Desarrollo Económico), con el objetivo de fortalecer la formulación e implementación de políticas públicas, la generación de productos de alto valor agregado, la producción de nuevos conocimientos a través de la investigación, la existencia de capital humano con habilidades técnicas y científicas y la habilidad para importar y adaptar el conocimiento producido fuera del país.

El Sistema de Información y Documentación Científica y Tecnológica de Recursos Humanos, Materiales de Organización y Financieros, "SIEDCyT" es un portal que integra la base para la generación de productos y servicios tecnológicos, en el estado de Baja California. 
Los insumos del sistema son: Instituciones de educación superior, centros de investigación, investigaciones realizadas, la infraestructura dedicada, las publicaciones científicas y de divulgación, así como los recursos disponibles para esta actividad en el estado de Baja California; lo cual nos proporcionará una estadística de la actividad científica y tecnológica, así como los recursos físicos y económicos con los que cuenta el estado para impulsar el desarrollo de esta actividad tan importante para esta región.

EL portal SIEDCyT proporciona la siguiente información:

a) Acceso directo al programa estatal del COCYT.

b) Difusión de los servicios que ofrece el Consejo Estatal.

c) Módulo de registro de instituciones y centros de Investigación, investigadores y grupos de investigación.

d) El sistema integra diferentes catálogos de información.

e) Concentrado del gasto estatal en investigación y desarrollo experimental, educación científica y tecnológica y servicios científicos y tecnológicos en el Estado.

f) Reporte de los indicadores de formación de recursos humanos especializados y de innovación.

El Sistema de Información y Documentación Científica y Tecnológica de Recursos Humanos, Materiales de Organización y Financieros, "SIEDCyT" se encuentra en la siguiente dirección electrónica: http://siedcyt.cocytbc.mx.

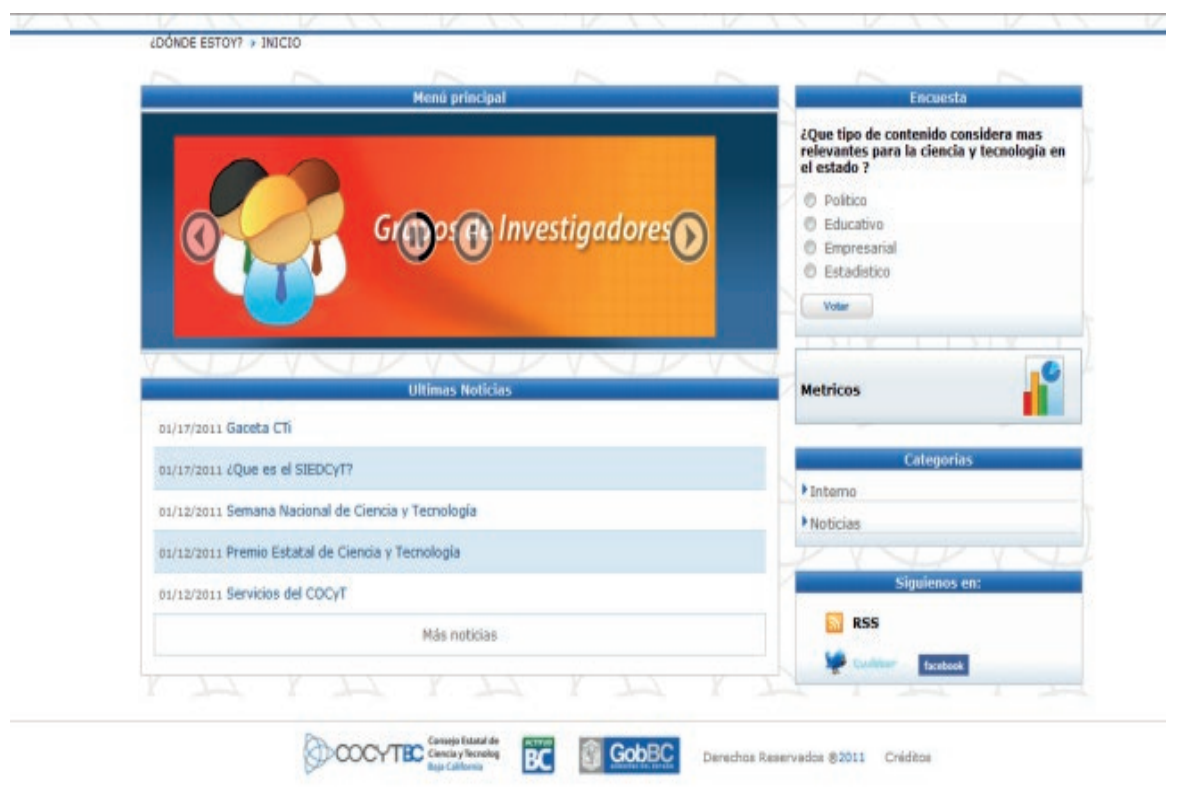

Figura 1. Pantalla Principal SIEDCyT. Fuente: COCYTBC 
La divulgación y promoción de la ciencia es de vital importancia para la generación de productos, servicios tecnológicos y estrategias, el sistema SIEDCyT tiene también como objetivo difundir información acerca de la vinculación y las formas en que se realiza esta, de modo que sea útil para conocer la situación que prevalece en el estado de Baja California respecto a esta actividad; también está claro que entre la ciencia, la tecnología, y la sociedad existe una estrecha relación, es decir, en nuestros tiempos la sociedad está tan ligada con estos dos factores que es imposible separarlos, por todo lo anterior este portal cuenta con 4 entidades básicamente.

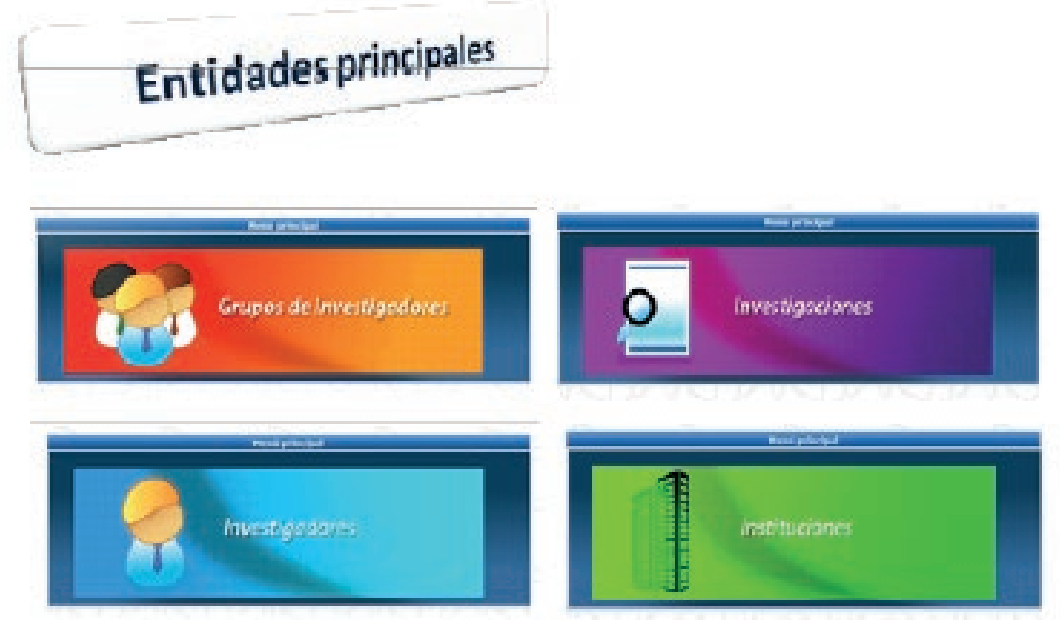

Figura 2. Pantalla de Entidades del Sistema SIEDCyT. Fuente: COCYTBC

\subsection{Investigadores}

El modulo de Investigador permite la definición de todos los datos de un Investigador, perfil PROMEP, perfil SIN, líneas de investigación, producción editorial, administración de grados académicos, participación en artículos en común, también permite crear los diferentes vínculos entre investigadores, dado que un Cuerpo Académico puede tener varios investigadores.

\subsection{Grupo de Investigadores}

Este modulo permite la definición de todos los datos de un conjunto de investigadores con una línea de investigación en común, también permite crear diferentes vínculos entre los investigadores pertenecientes a esta línea, la funcionalidad de este modulo permite la utilización de componentes para asociar proyectos de investigación y otros a este Cuerpo Académico.

\subsection{Instituciones}

El modulo de Instituciones permite la definición de todos los atributos de una Institución en general, permite crear diferentes vínculos entre Investigadores y cuerpos Académicos, dado que una Institución como tal puede contener varios 


\subsection{Investigaciones}

El modulo de Investigaciones nos permite identificar cuáles son las investigaciones realizadas en el estado de Baja California, nombre de la investigación, fecha de inicio y fecha de termino, Instituciones involucradas y status de la misma.

\section{ANÁLISIS Y DISCUSIÓN}

\section{Vinculación}

La vinculación es un tema de relevancia significativa para lograr niveles sobresalientes de Innovación y desarrollo en Ciencia y Tecnología para una región. La vinculación, es una actividad de suma importancia en la educación superior que se da mediante la interrelación con los sectores empresariales, para que en conjunto, aplicando el conocimiento, realicen proyectos que cubran las necesidades y demandas de la sociedad dando como resultado un impacto económico, social y tecnológico relevante.

Actualmente en el Estado de Baja California algunas de las formas en las que se muestra la vinculación en los principales centros de investigación e instituciones de educación superior son por medio de: Practicas profesionales, utilización de infraestructura, estancias de investigadores en otras instituciones de educación superior, estancias de investigadores con empresas, estancias de investigadores en organismos gubernamentales, consultorías y asesorías u otro.

La información que nos arrojo el proyecto de investigación SIEDCyT se pudo notar que las principales instituciones con las que se vinculan los investigadores para ejecución de proyectos conjuntos son: Instituciones académicas regionales $\mathrm{o}$ nacionales, Instituciones académicas internacionales, sector empresarial, instancias del Gobierno Federal, instancias del gobierno Estatal, instancias del gobierno Municipal u otras organizaciones no gubernamentales.

La vinculación se logra gracias al esfuerzo de colaboración entre investigadores docentes en proyectos específicos, con la finalidad de beneficiar a la sociedad cubriendo sus necesidades y demandas. En la actualidad existen programas de apoyo a la vinculación, en Baja California el COCYTBC “Consejo Nacional de Ciencia y Tecnología de Baja California" junto con el Gobierno Federal y Estatal ofrecen diversos programas para impulsar esta actividad, dichos apoyos son otorgados principalmente a investigadores pertenecientes al Sistema Nacional de Investigadores. 
En el proyecto de investigación SIEDCyT se conto con la participación de aproximadamente 300 investigadores con grado SNI, de un total de 508 vigentes en el sistema hasta el 2010. Para obtener dicha información se aplicaron cuestionarios, de los cuales se tomo la muestra para realizar el análisis referente a las principales instituciones con las que se vinculan los investigadores.

Las principales instituciones donde se concentran la mayoría de los investigadores son en CICESE, UABC, UNAM y COLEF en Baja California.

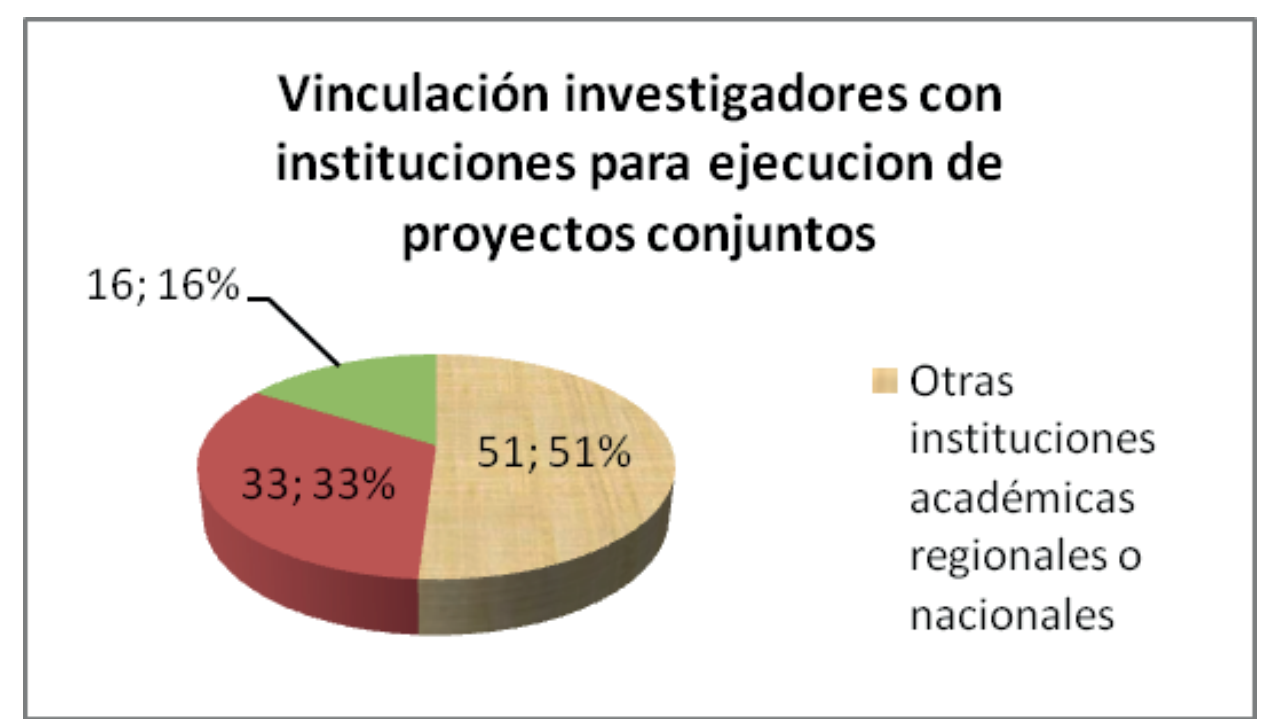

Figura 3. Vinculación para ejecución de proyectos conjuntos.

Fuente:SIEDCyT

Como se observa en la grafica anterior la vinculación para ejecución de proyectos conjuntos, se puede apreciar que un $51 \%$ de los investigadores pertenecientes al SNI realizan proyectos conjuntos con instituciones académicas regionales o nacionales, un $33 \%$ con instituciones académicas internacionales y el 16\% restante con el sector empresarial.

Esto refleja que más de la mitad de los investigadores hace vinculación con otras instituciones académicas regionales o nacionales como son: CICESE, UABC y UNAM. Las principales instituciones internacionales con las que se vinculan son: Universidades de California y Universidad Estatal de San Diego en Estados Unidos, Universidad de Alberta en Canadá, Universidad Politécnica de Cataluña en Barcelona, Universidad de Zaragoza en España, Universidad J. Fourier de Grenoble en Francia entre otras. 
Los principales motivos para establecer convenios de vinculación son:

a) Proyectos de colaboración para realizar investigación

b) Intercambio de infraestructura y compatibilidad de temas

c) Movilidad de estudiantes

d) Intereses académicos y de investigación afines.

La mayor parte de las actividades de vinculación con el sector empresarial se encuentran en la ciudad de Ensenada B.C., haciendo vínculos con el sector pesquero, ya que es una de las fuentes económicas más importantes para la ciudad, en Tijuana y Mexicali la principal forma en la que se muestra la vinculación, es mediante las prácticas profesionales que realizan los alumnos en diferentes empresas $u$ organizaciones gubernamentales. La actividad de vinculación es mínima por parte de los investigadores docentes con el sector empresarial.

Así como se necesita de una vinculación entre, docentes, investigadores y empresas, se deben desarrollar esfuerzos de innovación. Este esfuerzo innovador debe de ser una decisión fundamental que toda empresa debe de tomar en cuenta e incluir en su análisis estratégico. La innovación es clave para que las empresas crezcan y se posicionen en un buen nivel de mercado. Aquella empresa que no tiene la cultura innovadora difícilmente podrá subsistir y será arrasada cuando menos se lo espere. Cualquier innovación, ya sea tecnológica, de procesos, de servicios, organizacionales, gerenciales, etc., tienen cabida en todas las organizaciones y deben desarrollarse en cuanto antes.

Es por eso que debe de fomentarse esta cultura innovadora, sobre todo en las PYMES ya que es la fuente de recursos de toda economía en un país. Una de las tareas que se deben de fomentar en todas las empresas, es buscar vincularse con instituciones educativas o centros de investigación para que el gobierno proporcione los recursos necesarios y se lleven a cabo proyectos de innovación en conjunto.

\section{CONCLUSIONES}

Actualmente el Sistema de Información SIEDCyT se encuentra en funcionamiento, estamos en espera del recibimiento que este haya tenido por la comunidad de Ciencia y Tecnología en el estado de Baja California, y las implicaciones de este sistema ante el desarrollo en esta área del estado.

En la información primaria que se obtuvo en el proceso de desarrollo del proyecto refleja que más del 50\% de los investigadores pertenecientes al SNI en el estado de Baja California, realizan vinculación con otras instituciones académicas regionales o nacionales y también con instituciones de tipo internacional. Los principales motivos de este tipo de vinculación son debido a que comparten intereses en común en temas de investigación, o por intercambios entre alumnos de las distintas universidades. 
Los datos nos muestran que la vinculación entre el sector empresarial y centros de investigación es baja en relación a la existente entre centros de investigación y educativos, algunos factores influyentes en este resultado podrían ser que el modelo educativo o los planes de estudio de las instituciones educativas no responden a las demandas reales del sector empresarial. Por otra parte el sector empresarial no incluye en sus proyectos ni iniciativas de desarrollo a la educación, que podrían ayudar en gran parte para adoptar nuevas estrategias y técnicas que den respuesta a sus actividades diarias.

Considerando que la innovación y el cambio técnico no son procesos lineales ni características de una empresa, sino el resultado de un ambiente, un entorno que promueve espacios con capacidad para generar, adoptar y difundir innovaciones. Un territorio innovador es un sistema productivo vinculado a una o varias actividades, en el que una buena parte de las empresas y organismos existentes realizan esfuerzos en el plano de la innovación tecnológica, incorporando mejoras en los diferentes procesos asociados a su cadena de valor y en los productos que ofrece. Por lo anterior consideramos que el Sistema de Información SIEDCyT es una acción concreta para avanzar en el camino de la innovación tecnológica.

\section{REFERENCIAS}

Baca Urbina, G. (2006). Formulación y evaluación de proyectos informáticos. México: McGraw Hill.

Bernal Torres, C. A. (2006). Metodología de la investigación: para administración, economía, humanidades y ciencias sociales. Pearson Educación.

Cabrero Mendoza, E. (2006). El diseño institucional de la politica de ciencia y tecnología en México. México: Universidad Nacional Autónoma de México.

Cohen Karen, D. (2009). Tecnologías de Información en los negocios. México: McGrawHill.

Gido, J. (2007). Administración exitosa de proyectos. Cengage Learning.

Hernández Sampieri, R. (2007). Fundamentos de metodología de la investigación. México: McGraw-Hill.

López García, X. (2006). Sistemas digitales de información. Pearson Prentice Hall.

O'brien James, A. (2006). Sistemas de información gerencial. México: McGraw-Hill.

Rodríguez Durán, A. (2002). Ciencia, tecnología y ambiente. International Thomson. 
Takayanagui, A. D. (2000). La universidad del futuro: relaciones entre la educación superior, la ciencia y la tecnología. México: UNAM.

Tamayo Tamayo, M. (2007). El proceso de la investigación científica: incluye evaluación y administración de proyectos de investigación. México: Limusa.

Schilling, A. M, (2008). Dirección estratégica de la innovación tecnológica. España: McGraw Hill.

Gómez López, L. (2001). Gestión para la supervivencia de Daewoo en España.

Revista Telos, 101: 22-23.

Compendio de revistas electrónicas nacionales. (2009). Recuperado el 16 de enero 2009, de http://bivir.uacj.mx/temas/secciones/temarevistasnacionales.htm

CONACYT. (2009). Revista electrónica de ciencia y desarrollo. Recuperado el 16 de enero 2009, de http://www.conacyt.mx/comunicacion/revista/index.html

CONACYT. (2009). Índice de Revistas Mexicanas de Investigación Científica y Tecnológica. Recuperado el 16 de enero 2009, de http://www.conacyt.mx/Indice/Index_Indice.html.

REDALYC. (2009). Red de revistas científicas de America latina y el Caribe, España Portugal. Recuperado el 16 de enero 2009, de http:/ / www.redalyc.com/

Revista electrónica innovación-tecnológico educativa. Recuperado el 16 de enero 2009, de http:/ /cv1.cpd.ua.es/catalogaxxi/C10067PPESII1/S127769/P127740NN1/INDEX.H TML.

\section{Hilda Beatriz Ramírez Moreno}

Grado de maestra en Ciencias en Ciencias de la Computación por el Instituto Tecnológico de Tijuana, Coordinadora de Área de Sistemas de Información de la carrera de L. Informática. Miembro colaborador del cuerpo Académico en Formación "Sistemas de Información y Gestión Empresarial", línea de generación "Tecnologías de la información y comunicación en la toma de decisiones", según clave UABC-CA154 de PROMEP. Supervisora del programa de Servicio social Adultos en Plenitud Aprendiendo Nuevas Tecnologías del 2009 a la fecha. Responsable de Proyecto de Investigación: “Estudio comparativo del 2008 al 2012 de la factibilidad de impartición de cursos utilizando Tecnologías de la Información en la FCA, UABC Tijuana, vigencia 2011-2 a 2012-01. 


\section{Margarita Ramírez Ramírez}

Grado de maestra en Desarrollo Humano, por la Universidad Iberoamericana del Noroeste, y la maestría en Ciencias en Ciencias de la Computación por Instituto Tecnológico de Tijuana. Subdirectora Académica de la Facultad de Contaduría y Administración. Fue presidenta de Academia Ciencias Administrativas, en la UABC, período 2007-2009. Profesora en la Facultad de Contaduría y Administración, de la Universidad Autónoma de Baja California, campus Tijuana desde 1990 a la fecha, así como profesora en el Instituto Tecnológico de Tijuana, a partir del año 1991. Es miembro del cuerpo fundador de Academia Nacional ANFECA, así como Certificación de Informática Administrativa por ANFECA, cuenta con Perfil PROMEP. Actualmente coordinadora de la Maestría en Tecnologías de la Información y la Comunicación en la Facultad de Contaduría y Administración. 